Mitteilungen der Österreichischen Geographischen Gesellschaft, 160. Jg., S. 351-359

(Annals of the Austrian Geographical Society, Vol. 160, pp. 351-359)

Wien (Vienna) 2018, https://doi.org/10.1553/moegg160s351

\title{
Berichte und Kleine Mitteilungen
}

REPORTS AND NOTES

\section{LÄNDERKUNDE ABGESCHAFFT? ZUR BEDEUTUNG DER REgIONALGEOGRAPHIE IM DEUTSCHEN SPRACHRAUM VOR UND 50 JAHRE NACH KIEL UNTER BESONDERER BERÜCKSICHTIGUNG DER GEOGRAPHISCHEN LATEINAMERIKANISTIK}

\section{Festvortrag zum 80. Geburtstag von Christoph Stadel}

\author{
Axel BORSDORF, Innsbruck*
}

\begin{abstract}
„Länder- und Landschaftskunde sind unwissenschaftlich, problemlos und verschleiern Konflikte; sie haben keinen aktuellen Bezug. Länder- und Landschaftskunde werden deshalb abgeschafft!“ So und ähnlich formulierten die Studierenden 1969 ihre Forderung nach Umorientierung der Geographie auf dem Deutschen Geographentag in Kiel. 2019 jährt sich dieses denkwürdige Ereignis zum 50. Mal, Anlass genug, am selben Ort vom 25. bis 30. September 2019 den Deutschen Kongress für Geographie stattfinden zu lassen und Bilanz zu ziehen.

Anlässlich der Vollendung des 80. Lebensjahres von Christoph STADEL, also eines der großen Regionalgeographen, ist es angebracht, über die Rolle der Regionalen Geographie im 20. und 21. Jahrhundert nachzudenken, so wie es Cesar CAVIEDES (2013) in einem beeindruckenden Plädoyer für den länderkundlich-integrativen Ansatz in der Festschrift für Christoph Stadel vor genau fünf Jahren getan hat. Der Titel seines Beitrags „Der Niedergang der Regionalen Studien“ zeigt den Grundtenor des prominenten Schülers von Wolfgang WeISCHET. Ich meine, dass CAVIEDES ein zu negatives Bild der Gegenwart und Zukunft gezeichnet hat, zumal er sein letztes Kapitel „Trübe Aussichten für die Geographie als Disziplin und die Berufschancen der Geographen“ betitelte. Dabei bezog er sich zu sehr auf sein Heimatland, die Vereinigten Staaten von Amerika; im deutschsprachigen Raum dagegen kann man wohl kaum von trüben Aussichten sprechen.

Das möchte ich im Folgenden begründen und will dabei früh beginnen und die Anfänge der Regionalgeographie einbeziehen. Ich tue dies aus der Sicht eines Geographen, der noch die alten Meister studiert hat und auch meint, dass unsere heutige Zitationskultur, fußend auf dem Internet, manches neu erfindet, was längst bekannt, aber eben nicht digital verfügbar ist. Im ersten Teil meiner Ausführungen halte ich mich eng an die Analyse von CAVIEDES, der sozusagen aus der Außensicht die Entwicklungen im deutschsprachigen Raum verfolgt hat. Aus Zeitgründen setze ich den Fokus auf die geographische Lateinamerikanistik, in der Christoph STADEL ja einer der wichtigsten Vertreter ist.
\end{abstract}

\footnotetext{
* Emer. O. Univ.-Prof. Dr. Axel Borsdorf, Institut für Geographie der Universität Innsbruck, Innrain 52, A-6020 Innsbruck; E-Mail: axel.borsdorf@uibk.ac.at
} 
Die Länderkunde hat eine lange Geschichte, gewissermaßen steht sie am Anfang der geographischen Erkenntnis, und diese war für alle frühen Kulturen bedeutend. So ging es den Geographen der Antike, deren Werke überliefert sind, um die Kenntnis des eigenen Lebensraums und anderer Länder sowie um die Orientierung darin. Im Mittelalter reduzierte sich das Interesse auf die Geographie des Heiligen Landes, in der frühen Neuzeit waren es vor allem Reiseberichte aus den neu entdeckten Weltgegenden, bis mit Alexander von Humbold ein Universalgelehrter u. a. auch der Regionalgeographie eine wissenschaftliche Begründung gab. Er setzte dies etwa am Beispiel von Mexiko und Kuba auch um. Die Verknüpfung natur- und sozialwissenschaftlicher sowie lebensweltlicher Erkenntnisse ist seine große Leistung.

Leider konnte sein Niveau in den folgenden Jahrzehnten nicht immer gehalten werden. Ich überspringe ein Jahrhundert, in dem die Länderkunde mit Alfred KIRCHHOFF, dem genialen Lehrer Hans STEFFENS, ein starke Formalisierung erfuhr. Sie wurde später fälschlicherweise als „Hettnersches Schema“ bekannt. Dem widersetzte sich Hans Spethmann mit seiner Dynamischen Länderkunde. Deren Spuren fanden sich später in der problemorientierten Länderkunde wieder. Der Geograph Gerhard SANDNER und der Kulturanthropologe Hanns-Albert STEger haben die meines Erachtens beste derartige Länderkunde (zu Lateinamerika) vorgelegt. Wenn wir sie heute zur Hand nehmen, wird aber deutlich, wie zeitgebunden und rasch überholt ein solcher Ansatz ist. Dagegen erfreut sich das „Kirchhoff-Hettnersche Schema“ noch immer großer Akzeptanz und ist u. a. die Basis aller länderkundlichen Wikipedia-Artikel.

Natürlich habe auch ich, als einer der ersten deutschsprachigen Wikipedia-Autoren, immer dieses Schema verwendet. Und wenn ich heute manchmal noch einen regionalen Beitrag zur Online-Enzyklopädie schreibe - das ist selten geworden, weil schon praktisch alles behandelt wurde -, greife ich immer noch zum alten „Hettnerschen Schema“. Soviel schon mal vorweg zur Forderung nach Abschaffung der Regionalen Geographie!

Wir überspringen die Zeit, in der die Regionalgeographie - vor allem in den Staaten, die sich große überseeische Territorien aneigneten - einen Schwerpunkt in der Kolonialgeographie fand. Immerhin entstand in der Spätzeit in Bordeaux die angesehene Zeitschrift Les Cahiers d'Outre-Mer, gegründet von Louis PaPy und Henri Enjalbert. Damit begann die Hoch-Zeit der französischen Geographie, die mit Pierre Monbeig, Maurice Le Lannou, Hervé Thery, Collin Delavaux, Olivier Dollfus, Romain Gaignard, Jean Borde und Roland Paskoff sehr großen Einfluss auf die Weiterentwicklung der geographischen Disziplin in Lateinamerika nahm.

Nicht viel anders sah es mit der Lateinamerikanistik in Deutschland aus. Seit Alexander von HumBOLDTS Reisen in Lateinamerika zu Beginn des 19. Jahrhunderts galt die deutsche Geographie als besonders fortschrittlich. Den Spuren des großen Forschers folgten deutsche Reisende und Naturwissenschaftler auf den wenig bekannten Kontinent. Sie sicherten ihrem Heimatland Respekt und Bewunderung. Im späten 19. Jahrhundert schärfte der junge Alfred HetTNER sein Beobachtungs- und Interpretationsgeschick in Panama, Kolumbien, Peru, Chile, Argentinien und Südbrasilien. Die deutsche Geographie trug zwischen 1870 und 1920 seinen prägenden Stempel. HeTTNER erreichte den Höhepunkt seines Schaffens an der Universität Heidelberg, an der er prominente Lateinamerikanisten wie Oskar SCHMIEDER und Leo WAIBEL betreute. Diese bildeten dann ihrerseits eine weitere Generation aus.

Alle Vertreter der Regionalgeographie mit Blickrichtung auf Lateinamerika praktizierten eine Geographie mit Betonung auf Landschaftsentwicklung, Lebensweise und ethno-kulturellen Wurzeln der Bewohner. Enge Kontakte wurden mit Carl Ortwin SAuER gepflegt, der in den 1920er Jahren als Leiter des Department of Geography an der University of Berkeley, California, ähnliche Schwerpunkte setzte. Bei ihm sammelten u. a. die deutschen Junggeographen Oskar Schmieder, Gottfried PFeIFFER und Fritz BARTZ Auslandserfahrungen.

Nach der Ruptur des Zweiten Weltkriegs hatten die deutschen Universitäten erst in den 1950er Jahren wieder Geld für Forschung im Ausland und Stipendiaten aus Lateinamerika. Elisabeth 
LichteNBERGER (1984) hat darauf hingewiesen, wie stark - im Unterschied zu Österreich - die Forschungsförderung für Auslandsstudien in Deutschland war. Die Auslandsforschung wurde auch dadurch erleichtert, dass von den jungen Studentinnen und Studenten verlangt wurde, zumindest drei europäische Sprachen in Wort und Schrift zu beherrschen. Dies galt zumindest noch 1968, als ich mein Studium der Geographie in Göttingen begann.

Hervorragend in der lateinamerikanischen Regionalgeographie war damals die Universität Bonn, an der Carl Troll eine ganze Reihe von später führenden Lateinamerikanisten ausbildete. Schon vor dem Krieg hatte Troll Feldstudien in den Anden Südamerikas betrieben. Seine von Ingeborg MonHeIM redigierten und posthum publizierten Tagebücher der Reisen in Bolivien 1926/1927 zeugen von den erstaunlich tiefen Kenntnissen und der exakten Beobachtungs- und genialen Interpretationsgabe des jungen Troll (Monheim 1985). Nach dem Krieg führte er seine regionalen Studien dort fort und konzentrierte sie auf den westlichen Teil des Kontinents, die Andenländer. Diese blieben auch der Fokus seines Schülers Wilhelm LAUER, der nach ihm die Institutsleitung in Bonn übernahm. TROLL und LAUER schickten viele ihrer Dissertanten zur Feldarbeit nach Bolivien, Chile, Argentinien und auch Mexiko.

Dem Troll-Doktoranden Wolfgang WeISCHET bot man im Jahr 1961 den Freiburger Lehrstuhl von Nikolaus Creutzburg an, und damit war das dortige Geographische Institut auf dem Weg zur Hochburg der Regionalen Südamerika-Geographie. Seine Länderkunde Chiles - er war zuvor Institutsleiter in Valdivia und Santiago de Chile gewesen - erschien 1970, auf dem Höhepunkt der Kritik an der Länderkunde. Ich bin stolz darauf, die regionalgeographische Entwicklung Chiles vom Entwicklungsland (wie zur Zeit WeISCHETS) zum Schwellenland in einer Neuauflage seiner Länderkunde zu dokumentieren. Weischets prominenteste Schüler Cesar CAVIEDES, Winfried ENDLICHER und Werner Mikus blieben dem regionalen Schwerpunkt Lateinamerika treu und lehrten an der University of Florida, der Humboldt Universität Berlin und der Universität Heidelberg.

Im Wettstreit mit Bonn und Freiburg stand das Geographische Institut der Universität Kiel unter der Leitung von Oskar SCHMIEDER. Ihm verdanken wir die meisterhaften Darstellungen der Alten und Neuen Welt $(1962,1969)$. SCHMIEDER erwarb seine kulturlandschaftliche Perspektive in den Vorkriegsjahren bei Carl O. SAUER in Berkeley. Seine Arbeiten waren so außergewöhnlich, dass sie ohne Verzug in Mexiko ins Spanische übersetzt wurden. Nach seiner Emeritierung nahm SCHMIEDER 1958-1959 noch einen Lehrauftrag an der Universidad de Chile wahr.

SChmieders Erbe lässt sich bei Helmut Blume - dem bekannten Karibik- und Mittel- und Nordamerikaspezialisten - weiterverfolgen sowie bei Herbert WiLHeLmY, der nach der Promotion bei SCHMithenNer in Leipzig Assistent von SCHMIEDER in Kiel wurde. Beide lehrten später in Tübingen.

In Kiel setzte der Bonner Geograph und LAUER-Schüler Jürgen BäHR die lateinamerikanistische Tradition fort. In seinen Arbeiten über regionalwirtschaftliche Themen - wie Stadtmodelle und Stadtausbreitung - benützte er auch quantitative Methoden. Sein Interesse galt später auch Südafrika. Er brachte zahlreiche Schüler auf Lehrstühle, darunter auch Rainer WEHRHAHN, der bis heute sein Werk in Kiel weiterführt.

Den Aufschwung Tübingens in der geographischen Lateinamerikanistik geht nicht auf HETTNER zurück, der dort nur kurz lehrte, sondern auf Herbert WiLHELMY. In den 1930er Jahren war dieser auf Geländeforschung in Argentinien und Paraguay. Dort erreichte ihn eine Einladung von Carl SAUER nach Berkeley, doch der Ausbruch des Zweiten Weltkriegs machte diese Pläne zunichte. Nach dem Krieg konnte WiLHELmY aber doch eine Gastprofessur in Berkeley wahrnehmen.

Kürzere Stationen in Kiel und Stuttgart bereiteten seine Berufung nach Tübingen vor. Die Mannigfaltigkeit seiner wissenschaftlichen Interessen fand ihren Niederschlag in Werken, die ins Englische, Spanische und sogar Japanische übersetzt wurden. Aus seiner Feder flossen regionale Abhandlungen - zu nennen sind die Länderkunden über die La Plata Länder und Bhutan, die Studien zu Welt und Umwelt der Maya oder - landschaftskundlich - über den Reisanbau in Südostasien. 
Wilhelmy verehrte Alexander von Humboldt, über den er auch mehrfach publizierte. Sein breites, nahezu humboldt'sches Interesse führte zu botanischen, geomorphologischen, klimageomorphologischen, agrargeographischen, wirtschafts- und stadtgeographischen Studien in der ganzen Welt. Der Untertitel „Wesen und Wandel“, den er dem ersten Band des zweibändigen Werkes Die Städte Südamerikas (Wilhelmy und Borsdorf 1984) gab, entspricht seinem Erkenntnisinteresse: Es ging ihm darum, Räume als Organismen aus natur- und kulturräumlichen Faktoren zu erfassen (Wesen im ersten Sinn) und dabei ihre Charakteristiken herauszuarbeiten (Wesen im zweiten Sinn).

Von WiLhelmys Schülern wurden viele Ordinarien und Professoren, darunter Wolfgang BRÜCHER, Ralph Jätzold, Hartmut LeSER, Armin Hüttermann und meine Wenigkeit, und er hatte auch großen Einfluss auf Eckhart EhLERs, Günther Schweizer, Adolf LeIDlmair und Klaus Rother. Sein Lehrstuhl wurde von dem Brasilienforscher Gerd KoHLHEPP übernommen, der an WiLHelmys Lateinamerika-Interessen anknüpfte und dessen Schüler Martin CoY in Innsbruck und Martina NeUBURGER in Hamburg Lehrstühle übernahmen.

Gerd KohlhepP war ein Schüler von Gottfried PFeIFFer in Heidelberg. Dieser begann im Jahr 1949 seine Tätigkeit an der Wirkungsstätte von Alfred HettNer. In PFeIFFER vereinten sich verschiedene Traditionen, die sich in der Regionalen Geographie durchgesetzt hatten. Er war nicht nur ein treuer Anhänger seines Doktorvaters Leo WAIBEL, sein Verständnis für Lateinamerika war auch durch vier Jahre Lehrtätigkeit in Berkeley geprägt, wohin er während der Hitlerzeit emigrieren musste. Während seines langen Wirkens in Heidelberg beschäftigte sich PFEIFFER dann - auf Anregung von WAIBEL - vornehmlich mit Brasilien. Aus seiner Schule stammen eine Reihe bekannter Lateinamerikanisten, u. a. Felix MonheIm, Albrecht KesSLER, Erdmann Gormsen und - wie schon erwähnt - Gerd KoHLHEPP.

In Hamburg lehrte Gerhard SANDNER, der mit seinen Werken über die Städte und zur Regionalgeographie Mittelamerikas sowie einer Länderkunde Lateinamerikas (gemeinsam mit Hanns-Albert STEGer 1970) bekannt wurde. Seine Schüler Jürgen OssENBrÜGGE und Beate RATTER führten seine Arbeiten dort und in der Karibik fort. Aus Hamburg stammt auch der Mittelamerikaspezialist Helmut NunN, der in Marburg Ordinarius wurde und dort mit Günter MerTins und Ekkehard BUCHHOFER vorübergehend einen Lateinamerikaschwerpunkt bildete. Eine gewisse Zeit war auch Mainz mit Erdmann GoRMSEN und Gerhard ABELE (später Innsbruck) auf Lateinamerika ausgerichtet.

Ohne die Verdienste anderer Geographischer Institute zu schmälern, so muss man doch Bonn, Kiel, Heidelberg, Marburg, Tübingen und Freiburg als die Hochburgen der geographischen Lateinamerikanistik der zweiten Hälfte des 20. Jahrhunderts kennzeichnen. An diesen Instituten wurde das regionale Paradigma während der Nachkriegsjahre endgültig formuliert. So befand sich 1969, im Jahr des Kieler Geographentages, die Regionale Geographie im deutschen Sprachraum in bester Verfassung. Eine umfassende Länderkunde zu schreiben, galt als Königsweg der Geographen.

Man kann sich vorstellen, wie fassungslos manche der älteren Geographen nach dem Aufstand der Jungen auf dem Kieler Geographentag waren. Die Geographische Rundschau hat einige Repliken nach 1969 publiziert, und es ist noch heute spannend zu lesen, wie hilflos die Argumentation z. B. Oskar SCHMIEDERS war.

Es kann kein Zweifel daran bestehen, dass die in Kiel geäußerte Kritik - im Stil der Studentenrevolte von 1968 - verbal überzogen, inhaltlich aber doch teilweise gerechtfertigt war. Ein Blick in die Länderkunde von Hans-Günter GIERLOFF-EMDEN über Mexiko (1970) zeigt das ganze Dilemma: Diese besteht fast ausschließlich aus Zitaten anderer, die unkritisch hintereinander gehängt ein Sammelsurium von Scheinwissen ergeben, unmöglich zu lesen, noch unmöglicher zu verstehen. Dieses Buch dokumentiert wie kein anderes den Tiefstand regionalgeographischer Arbeiten. 
Es griffe zu kurz, die Kieler Ereignisse lediglich als Ausfluss der 1968er Studentenrevolte zu interpretieren. In sie flossen Strömungen ein, die aus unterschiedlichen Quellen stammten. Der Tübinger „Positivismusstreit“ zwischen POPPER und ADORNo und ihren Anhängern hatte die Sensibilität für die Wissenschaftstheorie geschärft. Dietrich BARTELs hatte unter diesem Eindruck eine wissenschaftstheoretische Grundlegung der Geographie des Menschen versucht, die großen Einfluss hatte. Gerhard HaRD, ein Schüler von Carl Troll, hatte das Landschaftskonzept der Geographie zerpflückt. Mit André Kilchenmann, Gerhard Bahrenberg und Ernst Giese nahm die deutsche Geographie Entwicklungen aus Nordamerika auf. Dort bauten Brian J. L. BERRY und Walter ISARD mit quantitativen Methoden eine positivistisch angelegte Regional Science auf, die scheinbar objektiv alle subjektiven Elemente der Länderkunde auszuschalten in der Lage schien. Zugleich - und dem entgegengesetzt - entstand eine marxistische Variante der Geographie, deren prominentester Vertreter David HARVEY war. Deren Apologeten appellierten an die Verantwortung der Wissenschaftler für die Gesellschaft und wollten nur ,gesellschaftsrelevante“ Ergebnisse zulassen.

Die „Kieler Revolte“ fußte also auf ganz unterschiedlichen und teil gegensätzlichen theoretischen Annahmen, und dieses Faktum erschwerte auch alle bislang erschienenen Versuche, die Kieler Ereignisse rückblickend zu werten. Daran hat es freilich nicht gefehlt, stellvertretend sei das Themenheft der Zeitschrift Geographica Helvetica aus dem Jahr 2014 genannt. Wir dürfen gespannt sein, wie dies auf dem zweiten Kieler Geographentag im Jahr 2019 gelingen wird.

Dennoch war die „Kieler Revolte“ geographie-politisch außerordentlich wirksam. In der Folge zog sich unsere Disziplin zunehmend aus der integrativen Darstellung räumlicher Sachverhalte zurück und überließ dies anderen Wissenschaften, die dann im Gegenzug sogar einen ,,spatial turn “ hinlegten. Dies galt vor allem auch für die von Carl Troll begründete Geoökologie und die von Ernst NEEF theoretisch konzeptualisierte Landschaftsökologie, die nun von der Bio-Ökologie weitergeführt wurde. Und es betraf die Landschaftskunde, deren modellhaft-typisierender Ansatz nun fast zur Gänze ebenfalls von der Biologie übernommen wurde.

Die scheinbare Unvereinbarkeit von natur- und sozialwissenschaftlicher Blickweise führte an manchen Standorten zur Trennung Geographischer Institute und zur Neugründung von Physiogeographischen und Humangeographischen Departments, die zum Teil auch verschiedenen Fakultäten angehörten. Mancherorts ging der Auflösungsprozess noch weiter, und es entstanden aus Geographischen Instituten solche für Hydrogeographie, Geobotanik oder Kulturwissenschaft. An anderen Orten wurden geographische Lehrstühle durch Nicht-Geographen nachbesetzt.

Physio- und Humangeographen publizierten nun lieber in Journalen der Nachbardisziplinen, daher wurden manche traditionsreiche geographische Zeitschriften wie Petermanns Geographische Mitteilungen aufgegeben, andere wie die von Troll gegründete Geographische Zeitschrift oder die von Humboldt initiierte Fachzeitschrift Die ERDE gerieten in Existenzkrisen. Spezialisierung war das neue Paradigma der Geographie, und manchmal hatte man den Eindruck, als ob die Geographen dabei waren, ihre Nachbardisziplinen neu zu erfinden und sich selbst aufzugeben. Hartmut LESER (2004) hat das mit dem Bild eines sich aufblähenden Luftballons verglichen: Die Oberfläche wird immer größer, der Kern immer leerer, und schließlich zerplatzt er.

Nach dem Kieler Geographentag fehlte es nicht an Warnern. Darunter war auch der aufgehende Stern der neuen quantitativen Geographie, André KILCHENMANN, der seine Altersgenossen 1973 zum Umdenken aufrief (unpubliziertes Manuskript):

„Entgegen anderslautender Meinung einiger meiner jungen und sonst gleichgesinnten Kollegen bin ich (noch?) nicht überzeugt, dass das Thema ,Regionale Geographie ' nur noch wissenschaftshistorisch interessant ist. Ich habe vielmehr den Eindruck, dass zur Zeit des berühmt-berüchtigten Kieler Geographentags im Zuge einer ungestümen Ablehnung des ,Landschaftsbegriffs' das Kind (die, Regionale Geographie') mit dem Wasser ausgeschüttet 
worden ist. Ich kann heute oft feststellen, dass eine ,Regionale Geographie 'von der Gesellschaft, von der Öffentlichkeit zunehmend gefordert wird: in der Planung! Wenn z. B. der Schweizerische Bundesrat nach den Entwicklungsmöglichkeiten des alpinen Berggebietes fragt, wenn für irgendeine Region ein Entwicklungskonzept, für ein Katastrophengebiet (z. B. die Sahel-Region) ein Sanierungsplan gesucht wird, dann meine ich, ertönt, versteckt, der Ruf nach einer, Regionalen Geographie‘ als Entscheidungsgrundlage, als Entscheidungshilfe!"

Keineswegs wurden alle Institute der Geographie vom Änderungsdenken erfasst. In Österreich und der Schweiz zeigte der Kieler Geographentag keine gravierenden Folgen. Auch in Deutschland blieben die großen Institute in Bonn und Köln, die kleineren in Tübingen und Eichstätt und selbst das Kieler Institut weitgehend unbeeindruckt. Davon zeugen die Publikationsreihen Wissenschaftliche Länderkunden (Wissenschaftliche Buchgesellschaft) und Länderprofile (Klett-Verlag), in denen auch nach 1969 hochrangige Regionalgeographien erschienen. Herbert PopP hat in diesem Zusammenhang 1997 auch auf die Reiseführer-Literatur deutscher Geographen hingewiesen.

An den Instituten, die die Spezialisierung oder gar Trennung der Physio- und Humangeographie vollzogen, vollzog sich schleichend - zum Teil auch bedingt durch Fehlberufungen - ein gewisser Bedeutungsverlust, und an manchen Standorten wurden die Geographie-Institute gänzlich aufgelöst, so etwa in Regensburg, Mannheim oder Stuttgart. Dort zerplatzte sozusagen der Luftballon, um das Bild von Hartmut LESER noch einmal zu bemühen.

Die Ächtung der Regionalen Geographie, die nicht nur die Länderkunde, sondern in noch stärkerem Maß auch die Landschaftskunde betraf, hinterließ eine gewisse Orientierungslosigkeit. In der Folge wurden gierig alle Anregungen und Ansätze aus Nachbardisziplinen und aus dem Ausland, vor allem dem englischsprachigen, aufgenommen und auf jedem Geographentag ein neuer ,turn “ verkündet. Da gab es den cultural turn, den social turn, den linguistic turn, den material turn, den spatial turn, den postcolonial turn, den interpretive turn und viele andere. Und ständig wurden neue Geographien erfunden: Die neue Kulturgeographie, die feministische Geographie, die neue Wirtschaftsgeographie, die neue Geographie des Wissens und andere. Der Paradigmenwechsel war selbst zum Paradigma geworden!

Attraktiv erschienen nun die Soziale und/oder Politische Ökologie und darin auch ihre marxistischen Lesarten. Der Alles-Erklärer Niklas Luhmann wurde mancherorts zum Guru. Ja, mit der These von Benno WerLEN „Geographie ohne Raum“ (1993) bestand sogar die Gefahr der Selbstaufgabe unseres Faches.

Es ist menschlich, dass in Existenzkrisen die Schuld gern bei anderen gesucht wird. Ich habe es oft selbst erlebt, dass den ,arglistigen“ Geologen, Ökologen, Biologen, Meteorologen, Soziologen, Kulturanthropologen etc. ein böser Wille nachgesagt wurde, wenn sie nach und nach von der Geographie aufgegebene Forschungsfelder besetzten und damit große Drittmittelprojekte einwarben. Die „Verdrängung“ fand dann sogar zuweilen im Wortsinn ,räumlich“ statt, wenn die mit den eingeworbenen Mitteln finanzierten Stellen in Dienstzimmern der Geographischen Institute untergebracht werden mussten. Der Erfolg der Nachbardisziplinen beruhte auch darauf, dass viele der von der Geographie aufgegriffenen neuen Theorien praxisfern blieben. Ihre empirische Umsetzbarkeit war schwierig und konnte internationale Forschungsförderungsinstitutionen kaum überzeugen.

Dennoch ergaben sich zu Beginn des 21. Jahrhunderts neue Perspektiven für die Geographie. Diese waren u.a.:

- die Chancen durch Nutzung neuer Technologien wie den Geographischen Informationssystemen und ihrer Verbindung mit Laserscanning-Techniken, aber auch anderer Informations- und Kommunikationstechnologien 
- die fortschreitende Entwicklung wirtschaftlicher und politischer Großsysteme, gemeinhin als Globalisierung bezeichnet

- das Bewusstwerden existenzbedrohender Risiken für die menschliche Zivilisation, also

- des anthropogen verursachten Klimawandels,

- des Anstiegs von Naturgefahren,

- der Ressourcenverknappung,

- der Risiken von Großtechnologien,

- der Zunahme von inner- und zwischenstaatlichen militanten Aggressionen, terroristischen Aktivitäten Religionskonflikten und

- der globalen Migrationsströme, bedingt durch die wirtschaftlichen Gegensätze zwischen den reichen Ländern des Nordens und den armen des Südens, durch Angriffs- und Bürgerkriege sowie den Klimawandel

Alle diese Herausforderungen können auch als Globaler Wandel bezeichnet werden. Sie sind mit der Natur-/Mensch-Beziehung verknüpft. Die dadurch für die Regionale Geographie erwachsenden Chancen wurden zuerst an den Standorten Bonn, Köln, Innsbruck und Graz erkannt, wo die Regionale Geographie ohnehin ihren Stellenwert behalten hatte. An diesen Instituten wurde um die Wieder- oder Neudefinition einer „Integrativen Geographie“ gerungen (MüLLER-MaHN und WARDENGA 2005). Der von Peter Weichinart (2005) als „Dritte Säule“ bezeichnete Weg bedeutete aber nichts anderes als die Rückbesinnung oder Verstärkung des integrativen Ansatzes der Geographie. Mensch und Natur, das sind miteinander eng verknüpfte Systeme. Das wussten schon Carl Troll, Josef SснмітнüsEN und andere, die aus dieser Erkenntnis die Landschaftsökologie und Integrationslehre entwickelten und beide Sichtweisen der Regionalen Geographie, die Choretik und die Synergetik, also die Länder- und die Landschaftskunde, befruchteten.

Hans STÖTTER und Martin COY, die den Standort Innsbruck zu einem Vorreiter der Integrativen Geographie machten, formulierten das im Rahmen der Einrichtung des Forschungsschwerpunkts „Globaler Wandel - regionale Nachhaltigkeit“" im Jahr 2007 so:

„Der Forschungsschwerpunkt soll sowohl dem für die wissenschaftliche Identität des Innsbrucker Instituts entscheidenden integrativen Fachverständnis der Geographie entsprechen als auch eine möglichst breite Integrationsbasis für die Weiterführung bisheriger Forschungsaktivitäten bieten. Mit diesen Überlegungen lehnt sich das Institut für Geographie an die aktuelle Diskussion innerhalb der Geographie zu neuen Möglichkeiten der Überbrückung der fachlichen Kluft zwischen Physischer und Humangeographie an. Dabei bedeutet die Einforderung der fachlich-inhaltlichen Integration keinesfalls die Rückkehr zu einer additiven Raumbetrachtung im Sinne eines „Reintegrationsmodells“, sondern ist von der Überzeugung getragen, dass jegliches Verständnis komplexer Phänomene und Prozesse unter den Vorzeichen des Globalen Wandels eine enge wissenschaftliche Auseinandersetzung mit den vielfältigen Wechselbeziehungen im Mensch-Umwelt-System voraussetzt. In diesem Sinne wird derzeit das sog. Modell der „Dritten Säule“ diskutiert, das die Integration durch eine neue hybride Geographie versucht und damit die Mensch-Umwelt-Beziehung, das quasi immanente Paradigma der Geographie, fachtheoretisch neu hinterfragt. "

In Innsbruck, der Alpenuniversität, fand sich mit Gebirgen ein Raumtyp, der mit seiner Sensibilität für Klimawandel und Naturgefahren, aber auch mit seiner wirtschaftlichen und kulturellen Kleinkammerung und Beharrungstendenz eine ideale Folie für die Anwendung des alten und nun wieder als neu erkannten Ansatzes bot. Die Alpen und die Anden waren dafür ideale Raumindividuen. Bald zog Graz nach, und als Thomas GLADE nach Wien berufen wurde, auch die Geographie der österreichischen Hauptstadt. In Salzburg hatte Peter WeichHart schon mit seiner Dissertation eine Neube- 
wertung des ökologischen Konzepts in Gang besetzt, zwischenzeitlich war er aber den Verlockungen anglophoner Theorien erlegen. Nach seiner Berufung nach Wien kehrte er zum integrativen Ansatz zurück und befruchtete den Innsbrucker Diskurs mit seinen Publikationen zur „Dritten Säule“.

Die integrative Geographie mit ihrem regionalspezifischen Ansatz wird in der Lehre nicht nur im Hörsaal gelehrt, sondern vor allem im Gelände auf Praktika und in kleinen und großen Exkursionen. Auf diese wurde in Österreich - im Unterschied zu anderen Standorten - nie verzichtet.

Für mich war es beglückend, in Salzburg mit Christoph STADEL einen kongenialen Partner und Freund zu finden, mit dem Studierende aus beiden Instituten nach Ecuador und Peru aufbrechen konnten. Dort konnten sie die alte und junge Regionalgeographie im lebendigen Raum erfahren und ihre Methoden anzuwenden. So haben wir in den publizierten Exkursionsberichten die Großlandschaften jeweils nach einer der bewährten regionalgeographischen Methoden zusammengefasst und diese dabei mit den Studierenden wiederholend eingeübt.

Christoph STADEL warb EU-Gelder für Projekte mit internationalen Kollegen in Kolumbien, Peru, Ecuador und Chile ein. Er ist ein Regionalgeograph alter Schule, der aufgrund seiner internationalen Erfahrungen in Deutschland, Österreich, der Schweiz, Kanada und dem Andenraum sowie wegen seinen vielfältigen internationalen Kontakten im anglo-, franko- und iberophonen Raum und seinen exzellenten Sprachkenntnissen wie kein anderer Geograph alle Fachentwicklungen konstruktiv-kritisch verfolgen konnte. Er konnte den Studierenden im Studium und vor allem für den späteren Beruf nicht nur Impulse, sondern auch wichtige Grundlagen vermitteln - im Sinne der oben zitierten Äußerung von André KILCHENMANN nach dem Kieler Geographentag.

So schließe ich denn mit einer Korrektur des pessimistischen Resümees von Cesar CAviedes. Nein, es gibt keine trüben Aussichten für die Geographie und die Berufschancen für Geographen, zumindest nicht im deutschsprachigen Raum. Die von ihm als „Pixel-Geographie“ gebrandmarkte Computerisierung und Virtualisierung wird in Innsbruck, Wien und Graz und sicher auch in Salzburg durch die praktische Anschauung im Raum und die intensive Auseinandersetzung mit den Wechselwirkungen im System Natur-Mensch ergänzt. Wir betrachten die neuen Techniken der Geographischen Informationssysteme, der Fernerkundung mit dem Laserscanning und der Informationsverarbeitung als zwar wichtige Hilfsmittel, die aber nie allein stehen können. Wir erkennen an, dass sie mit dazu geführt haben, dass die Regionale Geographie dabei ist, ihre Bedeutung zu festigen. Es scheint so, als ob Kiel überwunden ist.

\section{Literaturverzeichnis}

CAviedes C. (2013): Der Niedergang der Regionalen Studien. In: Borsdorf A. (Hrsg.): Forschen im Gebirge. Christoph Stadel zum 75. Geburtstag (= IGF-Forschungsberichte, 5). Wien, Verlag der Österreichischen Akademie der Wissenschaften, S. 157-171.

Gierloff-Emden H.-G. (1970): Mexico. Eine Landeskunde. Berlin, Walter de Gruyter.

LeSER H. (2004): Idee und Ansatz der Physiogeographie im Werk Herbert Wilhelmys. In: KoHLHEPP G. (Hrsg.): Herbert Wihelmy 1910-2003, Würdigung seines wissenschaftlichen Lebenswerks (= Tübinger Geographische Studien, 141). Tübingen, Geographisches Institut der Universität Tübingen, S. 43-84.

Lichtenberger E. (1984): The German-Speaking Countries. In: Johnston R. J., Claval P. (Hrsg.): Geography Since the $2^{\text {nd }}$ World War. An International Survey. London, Croom-Helm, S. 156-184.

Monheim I. (Hrsg.) (1985): Carl Troll. Tagebücher der Reisen in Bolivien 1926/1927. Stuttgart: Franz Steiner. 
Müller-Mahn D., Wardenga U. (Hrsg.) (2005): Möglichkeiten und Grenzen integrativer Forschungsansätze in Physischer Geographie und Humangeographie. Leipzig, Leibniz-Institut für Länderkunde (= forum ifl, 2).

Popp H. (1997): Reiseführer-Literatur und geographische Landeskunde. In: Geographische Rundschau 49, 3, S. 173-179.

Sandner G., Steger H.-A. (1970): Lateinamerika. Frankfurt Main, Fischer Taschenbuch Verlag (= Fischer Länderkunde, 7).

Schmieder O. (1962): Die Neue Welt. 2 Bände. Heidelberg - München, Keysersche Verlagsbuchhandlung.

Schmieder O. (1969): Die Alte Welt. 2 Bände. Kiel, Schmidt \& Klaunig.

Weichhart P. (2005): Auf der Suche nach der „dritten Säule“. Gibt es Wege von der Rhetorik zur Pragmatik? In: Müller-Mahn D., Wardenga U. (Hrsg.): Möglichkeiten und Grenzen integrativer Forschungsansätze in Physischer Geographie und Humangeographie (= forum ifl, 2). Leipzig, Leibniz-Institut für Länderkunde, S. 109-136.

Weischet W. (1970): Chile. Seine länderkundliche Individualität und Struktur. Darmstadt, Wissenschaftliche Buchgesellschaft.

WerLen B. (1993): Gibt es eine Geographie ohne Raum? Zum Verhältnis von traditioneller Geographie und zeitgenössischen Gesellschaften. In: Erdkunde. Archiv für wissenschaftliche Geographie, 47, 4, S. 241-255.

Wilhelmy H., Borsdorf A. (1984): Die Städte Südamerikas. Band I, Wesen und Wandel. Stuttgart - Berlin, Gebr. Bornträger. 